\title{
INTERACTION OF CONFIGURATION IN SPECTRAL OPACITY CALCULATIONS FOR STELLAR PHYSICS
}

\author{
D. Gilles ${ }^{1}$, S. Turck-Chièze ${ }^{1}$, M. Busquet ${ }^{2}$, F. Thais ${ }^{3}$, G. Loisel $^{4}$, \\ L. Piau ${ }^{1}$, J.E. Ducret ${ }^{1}$, T. Blenski ${ }^{3}$, M. Poirier ${ }^{3}$, C. Blancard ${ }^{5}$, \\ P. Cossé ${ }^{5}$, G. Faussurier ${ }^{5}$, F. Gilleron ${ }^{5}$, J.C. Pain ${ }^{5}$, J.A. Guzik ${ }^{6}$, \\ D.P. Kilcrease ${ }^{6}$, N.H. Magee ${ }^{6}$, J. Harris ${ }^{7}$, S. Bastiani-Ceccotti ${ }^{8}$, \\ F. Delahaye ${ }^{9}$ and C.J. Zeippen ${ }^{9}$
}

\begin{abstract}
We discuss the role of Configuration Interaction (CI) and the influence of the number of configurations taken into account in the calculations of nickel and iron spectral opacities provided by the OPAC international collaboration, including statistical approaches (SCO, CASSANDRA, STA), detailed accounting (OPAS, LEDCOP, OP, HULLAC-v9) or hybrid method (SCO-RCG). Opacity calculations are presented for a temperature $\mathrm{T}$ of $27.3 \mathrm{eV}$ and a density of $3.4 \mathrm{mg} / \mathrm{cm}^{3}$, conditions relevant for pulsating stellar envelopes.
\end{abstract}

\section{State of the art on Iron opacity comparisons}

Extensive comparisons of nickel and iron spectral opacity calculations have been performed by the OPAC international collaboration (Turck-Chièze et al. 2011a,b; Gilles et al. 2011). We have considered results obtained with the codes CASSANDRA (Crowley et al. 2009), STA (Busquet et al. 2010), OPAS (Blancard et al. 2012), LEDCOP (Magge et al. 1995), SCO-RCG (Porcherot et al. 2011),

\footnotetext{
1 SAp/IRFU/DSM, CE Saclay, 91191 Gif-sur-Yvette Cedex, France

2 ARTEP Ellicott City, MD 21042, USA

3 CEA/IRAMIS/SPAM, 91191 Gif-sur-Yvette, France

4 Present address: Sandia National Laboratories, Albuquerque, NM 87185-1196, USA

5 CEA/DAM/DIF, 91297 Arpajon, France

6 Theoretical Division, LANL, Los Alamos, NM 87545, USA

7 AWE Reading Berkshire, RG7 4PR, UK

8 LULI, École Polytechnique, 91128 Palaiseau, France

9 LERMA, UMR 8112, Observatoire de Paris, UPMC, CNRS, 5 place J. Janssen, 92195 Meudon, France
} 

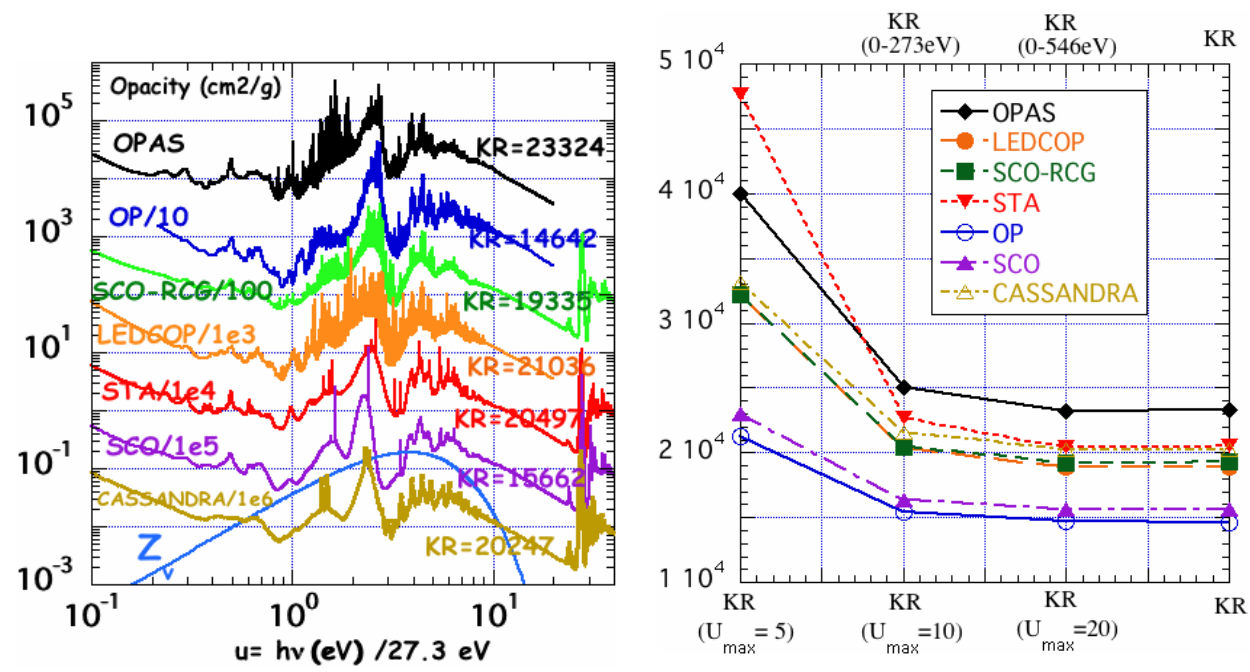

Fig. 1. (a) Iron opacity comparisons at $T=27.3 \mathrm{eV}$ and $\rho=3.4 \mathrm{mg} / \mathrm{cm}^{3}$, each spectrum is shifted by a decade for clarity; (b) Convergence of the corresponding Rosseland mean opacity coefficients $K_{R}$ versus the $U_{\max }, U=h \nu / k T$, values.

SCO (Blenski et al. 2000), OP (Badnell et al. 2005) for conditions where the study of spectra from ions with vacancies in the $\mathrm{M}$ shell $n=3 \rightarrow n=3,4,5,6 \ldots$ presents a challenge due to the very large number of transitions in the spectrum.

Figure 1a illustrates the large panel of different iron opacity results for $\mathrm{T}=27.3 \mathrm{eV}, \rho=3.4 \mathrm{mg} / \mathrm{cm}^{3}$, which corresponds to a mean ionization $\langle Z\rangle \approx 8$, equivalent conditions of the iron peak of stellar envelopes (Turck-Chièze et al. 2009, 2011; Gilles et al. 2011). The plasma is mainly composed of Fe IX and Fe X ions, with a few Fe VIII and Fe XI ions. Figure 1b illustrates the evolution of the Rosseland mean coefficient $K_{R}$ with the maximum value $U_{\text {max }}$ kept in the integral,

$$
\left[\frac{1}{K_{R}}\right]_{U \max }=\int_{0}^{U \max } \frac{Z_{\nu}}{K_{\nu}} d u, \quad Z_{\nu}=\left(15 / 4 \pi^{4}\right) u^{4} e^{-u} /\left(1-e^{-u}\right)^{2}, \quad u=\frac{h \nu}{k T}
$$

where $Z_{\nu}$ is the Rosseland weighting function, represented by the blue line on Figure 1a. All the features shown in Figure 1a in the range $0.5<u=h \nu / 27.3 \mathrm{eV}<$ 20 are of central importance in opacity calculations because they make a substantial contribution to the Rosseland mean opacities. Differences between Rosseland mean coefficient are directly related to this portion of the frequency-dependent opacities. At relatively low temperature and for the mentioned spectral range, bound-bound iron opacity contributions are dominant, mainly composed of $\Delta n=0, n=3$ and $n=3 \rightarrow n=4,5,6$ transitions for the Fe ions of interest. Consequently they must be included in opacity calculations to obtain consistent stellar model predictions. These transitions are very sensitive to the Configuration Interaction (CI) treatment thus the challenge is to treat altogether a large number of atomic excited levels in a "full" CI mode. 
In the present paper we discuss two important causes of differences between the theoretical spectra, the Configuration Interaction (CI) treatment and the number of atomic levels that are considered in the opacity calculation. Other effects here less important, Stark effect, for instance, being out of the scope of this paper.

\section{New comparisons including HULLAC-v9 calculations}

We have used new HULLAC-v9 calculations (Busquet et al. 2006, 2012) to discuss these two points. HULLAC is a flexible and powerful fine-structure relativistic code able to generate thousands of configurations and billions of lines and to compute LTE and non-LTE spectra. HULLAC and OP are the only calculations that introduce full CI, i.e. CI between all levels. But the number of levels to be treated in full CI mode often give dramatic increase of the computation time. The flexibility of HULLAC-v9 allows to see the role of different terms or CI approximations. In the full CI mode the computation of fine structure levels with CI (mode L for Levels) is chosen for defined groups of levels (GRL), see Busquet et al. (2012) for more details or illustrations. Selecting the CIinNRC mode, HULLAC-v9 computes the usual Relativistic CI (RCI) between all levels of one Non Relativistic Configuration (NRC). The diagonalization of the Hamiltonian matrix for all levels of same $\mathrm{J}$ is performed in a GRL. It is also possible to turn off the CI calculation using sub mode NoCI. Other GRL can be selected, like Layzer complexes (configurations with identical n-shell occupancies). In Figure 2a the different HULLAC-v9 mode: L full CI (diamonds), LnoCI (dots), CIinLAYZER (circles) and CIinNRC (dashes) are compared for the $\Delta n=0, n=3$ opacity results for which CI plays a very important role for the thermodynamical conditions of Figure 1a. We can observe that the main effect is a global red shift of the peak between 60 and $80 \mathrm{eV}$ and the disappearance of the structure around $40 \mathrm{eV}$. The same comparison with complete inclusion of $n=3 \rightarrow n=4$ transitions is under way.

In OP, it is not possible to include all excited levels of interest in the R-matrix formalism but complementary sets of levels treated with a structure code are taken into account. OP spectral opacity tables have required to limit the computation to the most contributing transitions only and this restriction is even more severe when using full CI because of the increased numerical complexity. Insufficient number of excited levels can explain why OP frequency dependent opacities show noticeable differences with other results displayed in Figure 1a as already discussed in Gilles et al. (2011). STA, OPAS, LEDCOP and SCO-RCG codes account for RCI (CIinNRC mode in HULLAC-v9). CI treatment is not included in CASSANDRA and SCO.

Figure $2 \mathrm{~b}$ shows comparison between detailed code OPAS (dotted line) and two HULLAC calculations using different choices of M-shell transitions (dashed line: $\Delta n=0, n=3$ and $n=3 \rightarrow n=4,5,6$ for Fe IX, Fe X, solid line: an other selection of the same group of transitions for Fe VII to Fe XI). Important features correspond to the inclusion of all these transitions. One can notice that the agreement would be quite good if all $\mathrm{M}$ shell transitions would be kept in HULLAC, but as CI is not totally included in OPAS, a small difference will nevertheless persist. 

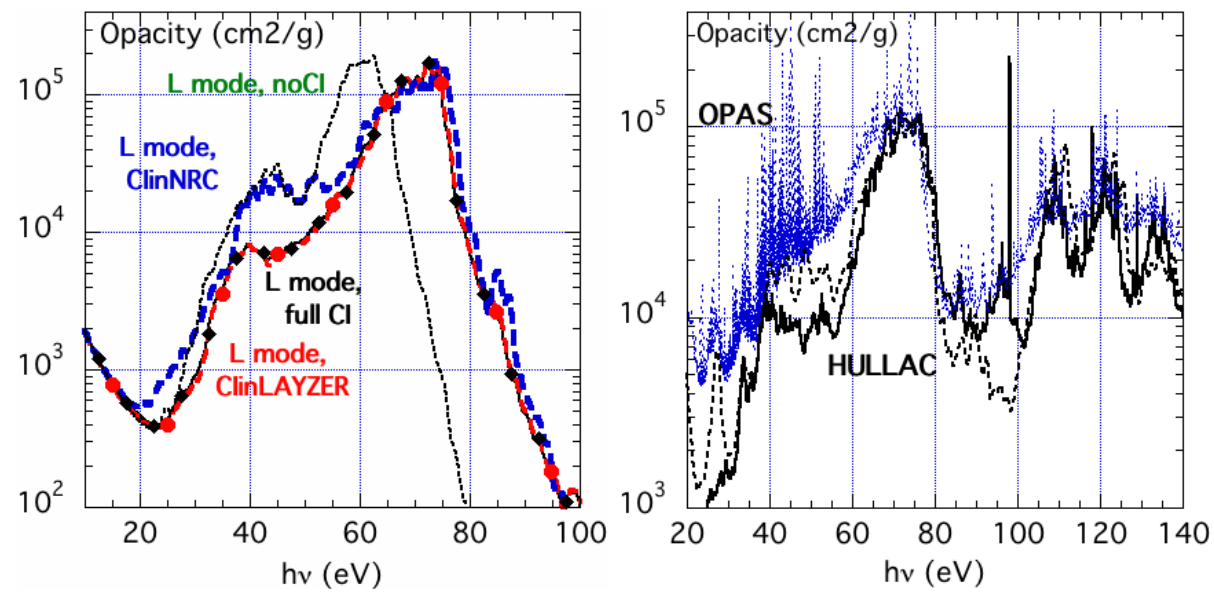

Fig. 2. (a) Four HULLAC-v9 CI mode iron opacity calculations (only $\Delta n=0, n=3$ transitions), (b) Comparison between OPAS and two HULLAC calculations (see text).

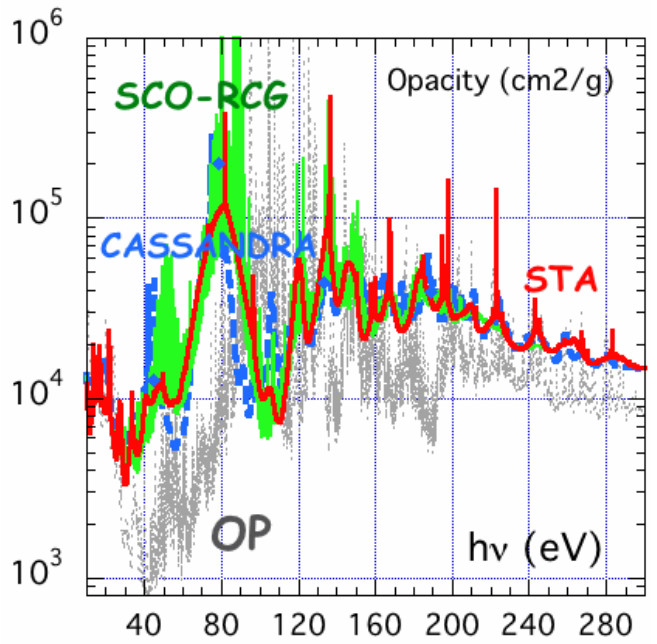

Fig. 3. Nickel opacity comparisons for $\mathrm{T}=27.3 \mathrm{eV}$ and $\rho=3.4 \mathrm{mg} / \mathrm{cm}^{3}$.

\section{Nickel opacity results and conclusion}

Differences in the nickel spectral opacity calculations obtained in the OPAC consortium have been already published for $\mathrm{T}=15.3 \mathrm{eV}, \rho=5.5 \mathrm{mg} / \mathrm{cm}^{3}$ (Gilles et al. 2011, 2012). The comparisons for $\mathrm{T}=27.3 \mathrm{eV}$ and $\rho=3.4 \mathrm{mg} / \mathrm{cm}^{3}$ are illustrated in Figure 3. Differences between OP and other opacity calculations are also important for these thermodynamical conditions. Comparisons within 
OPAC consortium and with HULLAC-v9 calculations are in progress. The interpretation of the discrepancies is still under study due to the dramatic increase of the computation time in HULLAC-v9 calculations with the number of levels to be treated in full CI mode.

\section{References}

Badnell, N.R., Bautista, M.A., Butler, K., et al., 2005, Mon. Notes R. Astron. Soc., 360, 458, and http://cdsweb.u-strasbg.fr/topbase/

Blancard, C., Cossé, P., \& Fausssurier, G., 2012, A\&A, 745, 10

Blenski, T., Grimaldi, A., \& Perrot, F., 2000, JQSRT, 65, 91

Busquet, M., Bar-Shalom, A., Klapisch, M., et al., 2006, J. Phys. IV (France), 133, 973

Busquet, M., 2010, Bull. Am. Phys. Soc., 55, 15

Busquet, M., Gilles, D., \& Klapisch, M., 2012, IFSA Proceedings, to be published

Crowley, B.J.B., \& Harris, J.W.O., 2009, JQSRT, 71, 257

Gilles, D., Turck-Chièze, S., Loisel, G., et al., 2011, HEDP, 7, 312

Gilles, D., Turck-Chièze, S., Busquet, M., et al., 2012, IFSA Proceedings, to be published Magee, N.H., Kilcrease, D., et al., 1995, ASP Conf., 78

Porcherot, Q., Pain, J.-C., Gilleron, F., et al., 2011, HEDP, 7, 234

Turck-Chièze, S., Delahaye, F., Gilles, D., et al., 2009, HEDP, 5, 132

Turck-Chièze, S., Loisel, G., Gilles, D., et al., 2011a, the OPAC consortium, Ap\&SS, 336,103

Turck-Chièze, S., Loisel, G., Gilles, D., et al., 2011b, J. Phys. Conf. Ser., 271, 012035 